\title{
An experimental environment for exchanging engineering design knowledge by cognitive agents
}

\author{
W. Shen, J.-P. A. Barthès \\ CNRS URA 817 HEUDIASYC \\ Université de Technologie de Compiègne \\ BP 649, 60206 COMPIEGNE, FRANCE \\ Tel: (33) 344234423 Fax: (33) 344234477 \\ E-mail: [Weiming.Shen IJean-Paul.Barthes]@utc.fr
}

\begin{abstract}
Large engineering design projects involve many different disciplines each with their own area of concern and expertise. A large amount of information (design data and knowledge) is processed and exchanged among such disciplines, and even within each discipline. Traditional computer environments cannot cope easily with such complex situations. Hence new approaches must be sought. This paper presents an experimental design environment organized as a population of asynchronous cognitive agents. Issues about the general architecture, the internal structure of an agent and inter-agent communication mechanism are discussed. A prototype including a number of independent agents is then presented and demonstrated on a small mechanical design.
\end{abstract}

Keywords
Cognitive Agents, Engineering Design Knowledge, CAD. Mechanical Design.

\section{INTRODUCTION}

Large engineering design projects require the cooperation of multidisciplinary design teams using sophisticated and powerful engineering tools such as commercial CAD tools, engineering databases, knowledge-based systems, simulation systems, or other special purpose computational tools. The individuals or the individual groups of the design teams work in parallel and independently, often for quite a long time, with tools located on different sites. Thus, at any time, individual members may be working on different versions of a design, viewing it from various perspectives (e.g., electronics, manufacturing, planning), at various levels of details. In order to coordinate the design activities of the groups and to guarantee a good cooperation among the engineering tools, it is necessary to develop efficient distributed (eventually intelligent) design environments. Such environments should not only automate individual tasks, like traditional computer-aided engineering tools, but also help individual members to share or exchange information (design data and knowledge) and to coordinate their actions as they explore alternatives in search of a globally optimal or near-optimal solution. 
Our approach to large engineering design projects is to decompose the overall project of designing a complex artefact into a set of different services. Such services are used by local teams which, in turn communicate with other teams. Typically such teams reside at different locations and are specialized in different aspects in the design of the product. Local teams work on a local network, and can also share or exchange design data and knowledge with remote teams via Internet. As a global policy, we do not plan to enforce a global consistency on the project. Each team needs a number of services, which are usually closely dependent on its particular engineering activities. Such services are to be assigned to agents both human and automatic.

Focusing on the work of a local set of specialists, we see the need for several types of services, which today are solved by various engineering tools. At this level of our approach, we found a number of other proposals aimed at using distributed problem solving techniques for addressing concurrent design. Indeed, in the past ten years, a number of researchers have proposed to use distributed problem solving technology for concurrent design (Gero 1987; Sriram et al 1991; Mantyla 1995); or proposed some general architectures or platforms for engineering or industrial applications such as SHADE (McGuire et al 1993), SHARE (Toye et al 1993), ARCHON (Jennings, Corera, and Laresgoiti 1995; Cockburn and Jennings 1995), SINE (Brown et al 1995), MIT DICE (Sriram et al 1989; Sriram et al 1992), DARPA DICE (Reddy et 1993; Londono et al 1992), KIEF (Tomiyama et al 1995), ICISs (Papazoglou et al 1992), Synchronous Group Applications (Tou et al 1994), PACO (Demazeau 1993) and OSACA (Scalabrin and Barthès 1993); or developed some agent-based systems such as PACT (Cutkosky et al 1993), First-Link (Park et al 1994), Next-Link (Petrie, Cutkosky, and Park 1994), Anarchy (Quadrel et al 1993), ACORN (Coyne et al 1994), ATOS-1 (Jones et al 1995), ABCDE (Balasubramanian and Norrie 1995), SiFA systems (Brown et al 1995; Dunskus et al 1995), CONDOR (Iffenecker 1994); some multi-expert systems such as DESIGN-KIT (Stephanopoulos et al 1987; Sriram et al 1989), IBDE (Fenves et al 1990), GUIDE (Morse 1990), CE Testbed (Brandt and Petro 1992; Londono et al 1992), MATE (Saad and Maher 1996), ANAXAGORE (Trousse 1993), EXPORT (Monceyron and Barthès 1992), ARCHIX (Thoraval and Mazas 1990), CAAD (Branki and Bridges 1993), some specific computer tools for inter-agent communication such as KQML (Finin, McKay, and Fritzson 1992), COOL (Barbuceanu and Fox 1995), ToolTalkTM (Frankel 1991), and (Populaire et al 1993), and some frameworks for inter-agent control (Van 1990; Lee, Mansfield, and Sheth 1993; Quadrel et al 1993; Boissier 1993).

The various approaches can be divided into two types of cooperative design systems according to their organization: multi-expert systems (based on a blackboard structure) and multi-agent systems. In a multi-expert system each specialist (usually called a knowledge source) has access to the blackboard where all information is posted and made available to all. In what we call a multi-agent system each agent is independent and has its own representation of the situation independent from that of other agents. In the first case, one normally uses a shared memory; in the second case, agents can be moved freely to independent machines, provided that they can communicate using messages. It is more than a simple difference in implementation.

Blackboard has been and still is a very popular architecture for building cooperative design systems. Several important design systems have been built using this architecture. IBDE (Fenves et al 1990) was established at CMU as a testbed for exploring integration of various specialists and the possible control strategies. GUIDE (Morse 1990) was also developed at CMU to test the problem of conflict resolution. KIEF (Tomiyama et al 1995) is a computational framework for knowledge intensive engineering developed at the University of Tokyo. It puts more emphasis on the knowledge acquisition problem. ANAXAGORE (Trousse 1993) was a joint project between the INRIA Research Institute and Aérospatiale. It involved the cooperation between a knowledge-based system and specialized CAD tools and was used to study multirepresentation and multi-configuration problems. ARCHIX (Thoraval and Mazas 1990) was a joint project developed at Renault, University of Paris VI, and University of Technology of Compiègne, to study the propose and revise strategy for designing front wheel drive-trains of cars. EXPORT (Monceyron and Barthès 1992) was a joint project between University of 
Technology of Compiègne and the Ministry of the Sea in France, and was implemented to integrate a number of existing tools used in the preliminary phase of harbour design. It included a number of specialists like breakwater design, residual wave motion computation, silt motion, etc. It emphasized the coupling of symbolic and numeric approaches. CAAD (Branki and Bridges 1993) was a joint project between Glasgow Caledonian University and University of Strathclyde in Scotland. It used a multi-blackboard architecture for distributed design environment. Such an architecture dispatches information over several blackboards, and each blackboard contains a relatively small amount of information. MIT DICE (Sriram et al 1992) is a complex blackboard system developed at MIT for studying cooperation processes among engineers. Recently, MIT DICE has been developed progressively through sub-projects like SHARED (Wong and Sriram 1993, Wong 1993), SHARED-DRIMS (Pena, Sriram, and Logcher 1993). The Concurrent Engineering Research Center (CERC) was established at the West Virginia University as a part of the DARPA Initiative in Concurrent Engineering (DICE) (Reddy et al 1993). CE Testbed was a main research result at CERC in order to demonstrate the capabilities and benefits of the DARPA DICE technologies (Londono et al 1990). A turbine blade engineering scenario has been implemented using the CE Testbed (Brandt and Petro 1992). MATE (Saad and Maher 1996) was developed at University of Sydney as a multi-user architecture for collaborative design in which existing applications, like CAD tools, modelling programs, analysis programs, knowledge-based systems can be shared by more than one designer. Two categories of shared workspace, shared visual representation and shared underlying representation, have been implemented in this project. Many other projects have also been developed or are being developed using a blackboard approach.

However, although a blackboard architecture has the definite advantage of ensuring consistency, it has nevertheless some drawbacks: (i) the blackboard can be a communication bottleneck; (ii) when using a blackboard to coordinate different engineering tools, each tool has its own representation of the data describing the problem to solve; such a representation is often private to the tool, and there is no particular advantage in transferring it to a centralized location; (iii) during a project lifetime new tools may be introduced, changing the structure of the design environment, and therefore the working environment has often to be reinitialized; (iv) the metaknowledge (knowledge about the project) is usually not recorded in the blackboard systems.

On the other hand, in multi-agent systems, each agent builds its own model of the current solution by acquiring information from the other agents. This type of system needs a communication protocol and a message format (common language) according to which the requests and the responses will be formed. Each agent stores the current solution, or at least a part of this solution in its local fact base. Generally, in multi-agent systems, agents are heterogeneous and autonomous, i.e., there is no global control, the communications among agents can be synchronous or asynchronous, point-to-point, broadcast, or multicast, and the messages among the agents have a high-level semantics.

Recently, some projects have been developed using an agent architecture. SHADE (McGuire et al 1994) is primarily concerned with the information sharing aspect of the concurrent engineering problem. SHADE demonstrated a flexible infrastructure for anticipated knowledgebased, machine-mediated collaboration between disparate engineering tools. It is a project within a larger cooperative community looking at related issues. PACT (Cutkosky et al 1993) was a landmark demonstration of both collaborative research efforts and agent-based technology. SHARE (Toye et al 1993) is concerned with developing open, heterogeneous, network-oriented environments for concurrent engineering. FIRST-LINK ( Park et al 1994) is a system of semi-autonomous agents helping specialists to work on one aspect of the design problem. NEXT-LINK (Petrie et al 1994) is a continuation of the previous project for testing agent coordination. All the previous systems were developed at Stanford. Anarchy, developed at CMU, (Quadrel et al 1994) is a working prototype of an asynchronous design environment for generating, evaluating and modifying designs of medium- and high-rise office buildings. The simulated annealing method was applied in Anarchy as a control strategy. ITX (Lee, Mansfield, and Sheth 1993) also addresses the issue of interaction control. SiFA (Brown et al 1995; Grecu and Brown 1995), developed at Worcester Polytechnic, is intended to address the issues of patterns of interaction, communication, and conflict resolution. Some interesting 
experiments were also conducted between CMU and Stanford University in particular by Finger in class projects with industrial problems (Reddy, Chan, and Finger 1995; Toye et al 1993).

We are currently developing a prototype of Distributed Intelligent Design Environment (DIDE) (Shen and Barthès 1995b; Barthès 1995) based on an architecture called OSACA (Open System for Asynchronous Cognitive Agents) (Scalabrin and Barthès 1993), derived from previous work in the domain of robotized assembly systems (Abriat 1991). Our goal is to verify whether it is actually possible to build truly open systems, that is, systems for which users can freely add or remove agents without having to halt or to reinitialize the work in progress in any way. We also want to exercise the obtained prototype in order to gain first hand experience, first with small examples, then with larger projects. Indeed, we are interested in fine in very large design projects of complex systems such as an automobile, a locomotive, a harbour, or an aircraft. Such design projects share the following characteristics: the design requires a large number of individual designers or several working groups to work together, the design period is long, and the design is very expensive. In this case, we do not think that a multi-expert architecture built upon a blackboard architecture could scale up. On the other hand, developing a network of independent local agents looks more promising. A major difference between our approach and that of SHADE-based projects (PACT, First-Link, Next-Link) is in its global structure. Indeed, in DIDE all agents are independent and first class; in particular, there is no facilitator structure like in PACT.

The rest of the paper describes our project. It is organized as follows: Section 2 presents the general DIDE architecture, the internal structure of a single agent, as well as the inter-agent communication. Section 3 describes the experimental implementation of the DIDE with a small mechanical design example. Section 4 gives some concluding remarks and discusses some important issues.

\section{A MULTI-AGENT ENVIRONMENT FOR COOPERATIVE ENGINEERING DESIGN}

\subsection{A general architecture for design environment}

The overall organization of an agent-based architecture for design was described in the introduction. Several issues need to be clarified.

The system is not intended to run automatically. On the contrary, human beings are part of the system. The system cannot be organized independently of the company structure. Thus, we assume the project will be run by a project manager, and that each local group will in turn have a local project manager. We are interested here in a local group. Within the local group, some agents will be controlled by humans, other will provide services automatically. When humans are in full control, then this amounts to a traditional client/server architecture. It becomes more interesting when some agents are actively offering services knowing the final goal, i.e., the agents not only reply to the requests of the other agents, but also can give some suggestions or advice to the current design project according to their knowledge.

A second issue relates to the control of the design task. Apart from the project manager we assume no central control of the design task. Subtasks are created for answering requests as needed, once the work is initiated. There is no global planning neither central nor distributed. From this point of view, the agents are purely reactive. However, some subtasks may have predefined sequences (e.g., scenarios, amounting to local plans). When this is the case, there is no reason why such sequences should not be used.

A third issue concerns the global consistency of the design. The consistency is kept at a minimum, considering that each agent has a local model of the designed device. However, in mechanical design it is sometimes possible to show a model of the designed product. In such a case, each subgroup is allowed to modify the model independently, using its own design space. Results are kept in different versions of a model database. Then, at each progress meeting, it is the responsibility of the product manager to merge all the version propositions into a unique 
design. Of course, the environment has to support the merging process reconciliation efficiently (Barthès 1993).

A fourth issue concerns the agent behaviour. We assume that all agents are connected by means of a network, local network or Internet. Each agent can reach any other active agent by means of a broadcasting message. All agents receive messages. They may or may not understand such messages. When they do not understand a message, they simply do nothing. Whenever they understand the message, then they start working on it, provided the priority of the message is higher than the current work they were doing. Thus, agents are multi-threaded. When a new agent is introduced, it is simply connected to the network. Thereafter, it receives messages like any other agents.

A fifth issue is related to the legacy problem. In our design environment, an agent offers some specific service, usually by encapsulating a particular engineering tool. The agent interaction relies on three things (Cutkosky et al 1993): shared concepts and terminology for communicating knowledge across disciplines, an interlingua for transferring knowledge among agents, and a communication and control language that enables agents to request information and services. This technology allows agents working on different aspects of a design to interact at the knowledge level, sharing and exchanging information about the design independently of the format in which the information is encoded internally.

\subsection{Internal structure of an agent}

In our environment, agents are autonomous cognitive entities, with deductive, storage, and communication capabilities. Autonomous in our case means that an agent can function independently from any other agent. Figure 1 shows the internal structure of an agent (Ribeiro Gouveia and Barthès 1993).

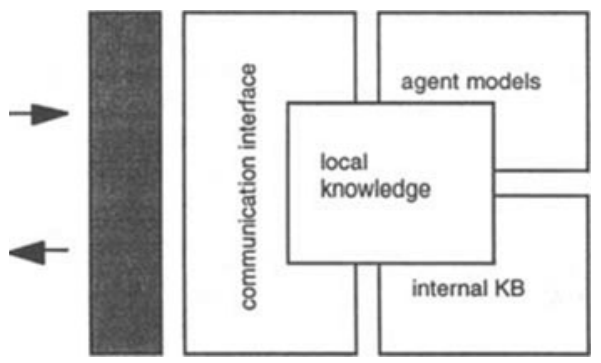

Figure 1 Internal structure of an agent in DIDE.

An agent is composed of: (i) a network interface (shaded portion in Figure 1); (ii) a communication interface; (iii) symbolic models of the other agents, and associated methods to use them (agent models in Figure 1); (iv) a model of its own expertise (internal KB); (v) a model of the task to be performed, or of the current context (local knowledge).

The network interface simply couples the agent to the network. The communication interface of an agent is composed of several methods or functions for sending and receiving messages to and from other agents. A message box is used to temporarily store all received messages. Processing incoming mails requires two steps: (i) receiving, storing, and sorting messages; (ii) encoding a message content for further processing by the agent in the context of a particular task. Processing out going mail similarly requires encoding of the information to be transmitted, and actually mailing it according to the exchange protocol. The symbolic models of the other agents are realized by means of a knowledge base containing information about the other agents, obtained during interaction. The information includes an agent's name, address, and skills or competences. The knowledge helps the current agent to select one or more agents as 
sub-contractors for processing tasks. The model of "own expertise" is also a knowledge base composed of self information such as the name, address and competences or skills. The latter may be methods for activating tasks corresponding to the received requests. The local knowledge is a knowledge base composed of the information about the global task to be performed. It contains some already achieved partial solutions for a particular task.

At first, when a new agent is connected to a group of active agents, then only its communication interface and its own expertise contain information. The part recording facts about the work to be done, or the capabilities of the other agents is empty. In the case of slave agents (e.g., a local database, Local DB, as described in Section 3), this will not change, i.e., it will remain empty. In other cases, each agent must build its own image of both the work to be done and the capabilities of the other active agents, by extracting information from the various messages it receives.

\subsection{Inter-agent communication}

In general, communication can be synchronous or asynchronous, and the communication mode can be point-to-point (between two agents), broadcast (one to all agents), or multicast ( to a selected group of agents). The OSACA-based DIDE environment uses a multi-cast mode which can be extended to point-to-point and broadcast. In addition, DIDE allows for five simple actions: request, inform, announce, bid and notice. They are grouped into two categories: requests and assertions (REQUEST, INFORM, NOTICE); call for bids and offers (ANNOUNCE, BID).

\subsection{Task structure}

Task execution is initiated locally and can be done in different manners. Firstly, agents can broadcast information to all the other agents and then wait until some agent has computed the answer. In this way, the task can be carried out by several specialists of the subject, working in parallel (efficient only when they reside on distinct machines). Secondly, a contract protocol can be used, i.e., an agent broadcasts an offer describing the job to be done, waits for some time for submissions from the other agents, and then awards the contract to a particular agent according to some local criteria. Thirdly, a contract can be allocated directly to a known specialist. Note that although the last solution could appear to be the most efficient, a general broadcast allows all the agents to see the content of the request. Therefore, even if agents are not directly concerned by the request, they can nevertheless use part of its content to update their internal representation of the task being done or of their image of the expertise of the requesting agent.

\section{IMPLEMENTATION}

A small system has been developed for testing the basic feasibility of the project, as well as the possibility to encapsulate traditional tools, and to bring agents in and out without halting the system.

The first version used only slave agents (no active agents) and looked like a generalized client/server architecture. The current version, still being developed, uses the OSACA platform. Although developed as a local group, i.e., using several workstations on a local network, it has the capability of intergroup communication using Internet.

\subsection{Hardware and software environment}

The experimental environment is built on a network of SUN and VAX workstations. The OSACA platform developed in the DAI research group of University of Technology of 
Compiègne (Scalabrin and Barthès 1993) is used for defining agents and inter-agent communications. Some agents are implemented as a MOSS environment (Barthès 1989), a system of recursive frames capable of modelling objects with versions and well adapted to design activities, constructed on top of Common LISP. Some other agents are developed in C. Two databases MATISSETM (an object-oriented database, commercial product of ADB) and EDBMS (an extended relational database developed at the Chinese Academy of Sciences) are used for storing design data and knowledge.

\subsection{Description of DIDE agents}

The current version of DIDE contains a number of agents, including Project manager, Monitor, Database of Engineering Standard, Object-Oriented Distributed Database, Graphical Tools and a group of Design Tools (as shown on Figure 2).

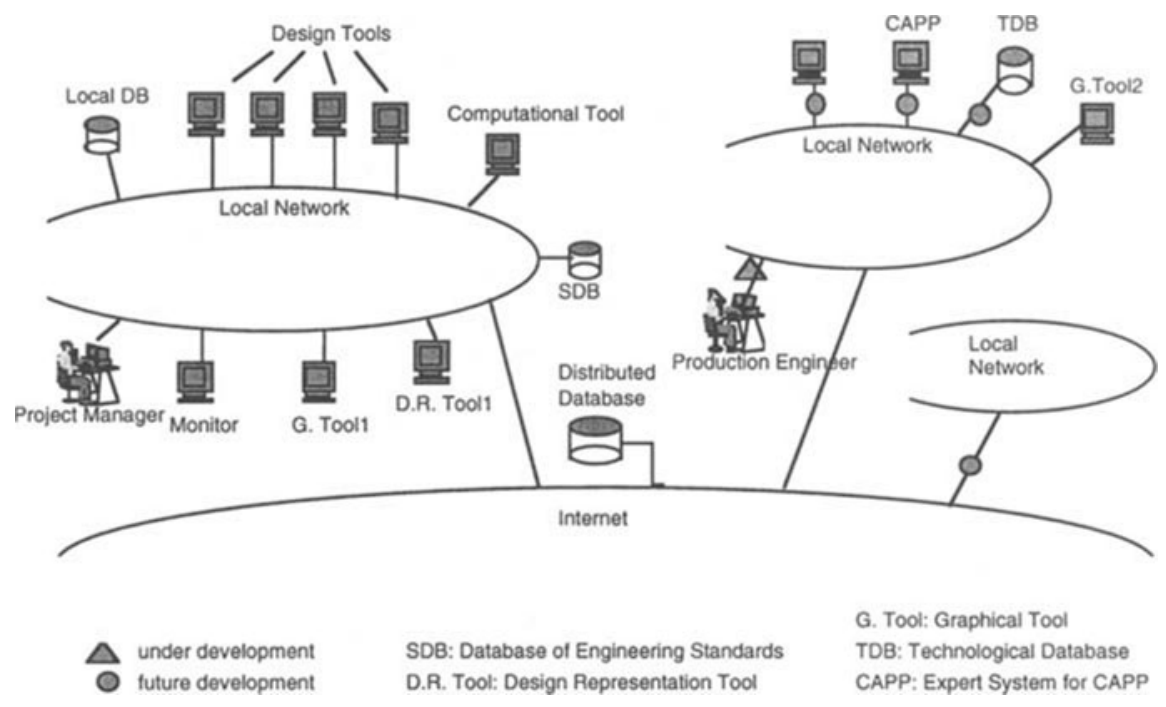

Figure 2 An experimental system architecture of DIDE.

Project Manager: The interaction mechanism for agents in DIDE has been developed to meet the needs of human designers working on a collaborative project. Here the agent Project Manager is taken as this type of "human" agent for specifying the initial parameters, some constraints for the design project, supervising the design process and resolving some conflicts detected by Gear Box Assembly agent. In the future DIDE environment, there will be several agents for human specialists from different design groups or from different domains such as dynamics, electronics, economics, and process engineering, for discussion and negotiation.

Monitor: This can be seen as an administrative agent. Generally, it works for the project manager. It receives all messages sent by all the other active agents in the same local system. All received messages are displayed on a text interface and a graphical interface is used to show the current system situation. When a new agent is connected to the system, an icon appears on the graphical interface, and when an old agent is removed from the system, the corresponding icon disappears. If there are some messages passing between agents, some signs appear on the graphical interface for a short time. At the same time, it modifies dynamically two HTML files: one for displaying the information about all active agents, another for showing the current 
situation of the design project. The two HTML files are accessible by all participants of the project via Internet. Finally, it saves also all messages into a log file.

Design Representation: This agent is responsible for the generation and management of geometric entities of a particular mechanical system. All classes, instances and methods are defined in this tool by using an object-oriented language, MOSS. The objects can be stored directly in a local extended relational database EDBMS or a distributed object-oriented database MATISSETM.

Computational Tool: This agent is responsible for engineering calculations. It may be a Finite Element Analysis tool, Optimizer or some other engineering computation tools. Currently it is a simulated Optimizer for gear box optimization.

Database of Engineering Standards: A database of national engineering standards is imperative in the domain of mechanical CAD. This agent is used for searching required standard dimensions in the database of engineering standards.

Following four Design agents are developed specially for an experimental design example, the Gear Box Design. The agent Gear Box Assembly is used for global design, assembling the parts, propagating constraints and detecting conflicts and resolving some of them automatically. Three other agents are used to carry out sub-tasks (parts design) in parallel. For large design projects, the agent Gear Box Assembly corresponds the global system design, and the other agents should be developed to accomplish the design of the sub-systems, or sub-sub-systems.

Gear Box Assembly: This agent is used to take the global design of the gear box. It decomposes the global design task into some sub-tasks and asks some special agents to accomplish the sub-tasks. Constraint propagation and verification is performed by this agent. Therefore, it is responsible for detecting conflicts by comparing the results obtained from the agents carrying out the sub-tasks. If some simple conflicts are detected, they may be resolved by some criteria in this agent. If not, a request message is sent to Project Manager to ask for a decision. At the end of a design or modification, the results can be shown on a 2D graphical interface, built on LispView.

Bolt Assembly Design: It is used to take the sub-task sent by Gear Box Assembly for completing bolt assembly design: standardizing bolts, nuts, washers. It shares mechanical objects with other concerned agents through a local database EDBMS or through Distributed OODB MATISSE.

Bearing Selection: It is used to take the sub-tasks sent by Gear Box Assembly for completing bearing selections.

Gear Design: This agent is used to take the sub-task sent by Gear Box Assembly for completing the big gear design. It also shares objects with other concerned agents through databases (local or distributed).

Graphical Tooll: Currently, AutoCAD ${ }^{\mathrm{TM}}$ on a SUNTM workstation with SOLARISTM 2.3 is used to display the design results. It can be also used to prepare a mechanical drawing or to let the drawings be modified by a human domain specialist. In this case, the agent is taken as a human agent and the main window of AutoCAD TM can be taken as a user interface for human specialists after some development on AutoLisp (This is not the case of current implementation, but is a future development). The data exchange with other agents is done by means of standard DXF (Drawing Interchange Format) and IGES files.

Graphical Tool2: This graphical tool is SDRCTM I-DEASTM located on the network of DECTM $^{\text {TM }}$ wrstations. In the current version of DIDE, this agent communicates with other agents by HTTP. This agent is used to test communications among agents located on different local networks and with different operating systems. The experimental goal is also to create graphical interfaces for production engineers to verify technological constraints.

Local $O O D B$ : An agent has been developed to encapsulate the extended relational database EDBMS for storing the mechanical objects shared by concerned agents in the local network.

Distributed OODB MATISSE: MATISSETM is a commercial object-oriented database of ADB. An agent has been created by using the kernel system of MATISSETM in the OSACA project for storing MOSS objects created and used by all OSACA-based agents. In DIDE, we 
use it as a second potential database for storing the mechanical objects shared by concerned agents.

\subsection{Inter-agent messages}

In the current DIDE implementation, only three types of messages (REQUEST, INFORM, NOTICE) are used for inter-agent communications. REQUEST messages can be sent by a method =request, and answers can be obtained by a method =get-result, e.g.,

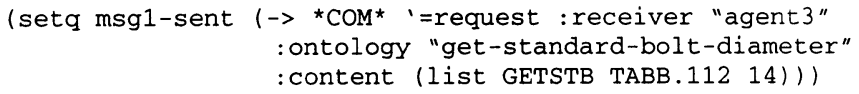

INFORM messages can be sent in the same fashion by a method =reply or by a method =inform with an option : in-reply-to. And NOTICE messages can be sent in the same fashion by a method =inform but without the option : in-reply-to. Before being sent out, messages are filled with some default values like : sender, :id, or : language by the current agent.

Here are some examples of the three types of messages (traces).

agent6:

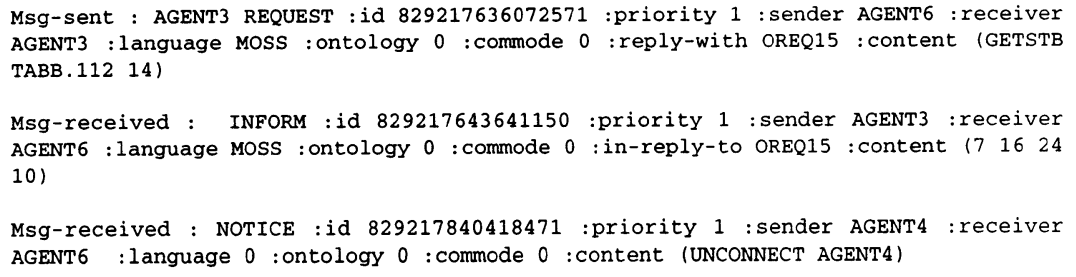

\subsection{Agent models}

As mentioned in Section 2, a model of agent including the symbolic models of other agents and the model of its own expertise is very important for cognitive agents. In the experimental DIDE environment, the "model of own expertise" for an agent is a simple knowledge base composed of the information about itself such as its name, address and competences or skills which may be some methods for activating some actions corresponding to the received requests. This simple knowledge base is stored as a MOSS object, instance of the class KNOWLEDGE:

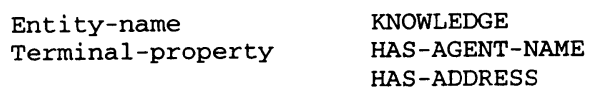




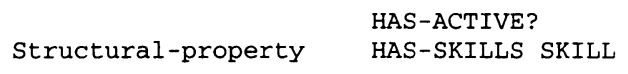

where the class SKILL:

$\begin{array}{ll}\text { Entity-name } & \text { SKILL } \\ \text { Terminal-property } & \text { HAS-SKILL-NAME } \\ & \text { HAS-KEY-WORDS } \\ & \text { HAS-EXPLANATIONS }\end{array}$

As a simple example of the above mentioned agent Computational Tool:

$\begin{array}{ll}\text { HAS-AGENT-NAME } & \text { agent } 7 \\ \text { HAS-ADDRESS } & \text { rouky.hds.utc.fr } \\ \text { HAS-ACTIVE? } & \text { yes } \\ \text { HAS-SKILLS } & \text { skill.1 }\end{array}$

where skill.1

$\begin{array}{ll}\text { HAS-SKILL-NAME } & \text { OPTIMIZE } \\ \text { HAS-KEY-WORDS } & \text { gear-box-optimization } \\ \text { HAS-EXPLANATIONS } & \text { "Optimize the main parameters of a gear box" }\end{array}$

The symbolic models of other agents are implemented by means of a knowledge base containing information about other agents obtained during interaction with the other agents. The information includes the agent's name, address, and its skills or competences. The same object structure is used to store this information about other agents. The knowledge helps the current agent to select one or more agents as sub-contractors for processing actions. The current message passing mechanism for obtaining this information is as follows: as soon as a new agent connects to the system, it broadcasts a message of REQUEST type and with context "REGISTER" to all other agents. Each one of the other agents receives this message, extracts the information of interest concerning the new agent such as its name, address and skills, and uses it to update its agent models, and then sends an "INFORM" message to the new agent. The new agent receives "INFORM" messages from all other agents, extracts important information and uses it to update its agent models.

The task model is created and updated by each agent during its interaction with other agents. From receiving and sending messages, it extracts the important information's such as the project name, sub-task names, situation of the each sub-task and the time estimated for a subtask or the time used for a completed sub-task. It saves also some important traces such as the content of the sending message and the results received from other agents for some REQUEST messages. In this fashion, when an agent sends a REQUEST message, it first verifies whether it sent a similar REQUEST previously and obtained a result. If so, it does not send the REQUEST message over again, but gets the results directly from its task model.

\subsection{A scenario - a small example}

As a design example, we considered a small mechanical system: a simple gear box, already described in details in (Shen and Barthès 1995a). Supposing that the necessary DIDE agents have all been connected to the system and each agent has established its agent models by message passing as mentioned in Section 3.4.

In the current implementation, only three types of messages (REQUEST, INFORM and NOTICE) are used. This paragraph shows how an agent handles such messages (see Figure 3). When an agent receives a REQUEST message for some sub-task, this agent chooses a method according its internal KB (knowledge about itself), executes this method for completing the sub-task, and then sends the results by an INFORM message to the sender (agent) of the 
REQUEST message. During the execution of the method, this agent may have to ask some other agents to complete part of the sub-task (sub-sub-task). It chooses an agent B according to its agent models (knowledge about other agents) and sends a REQUEST message to the agent B. After receiving the results by INFORM message sent by agent B, the agent resumes its work. During the work, some important information is saved in its local knowledge base for future use. A more complicated mechanism has been used to select an agent for carrying out a sub-task or sub-sub-task, which was described in details in (Shen and Barthès 1995b). A NOTICE message is similar to a REQUEST message, but when an agent sends a NOTICE message, it does not wait for a response; and when an agent receives a NOTICE message, it processes this message without sending any response.

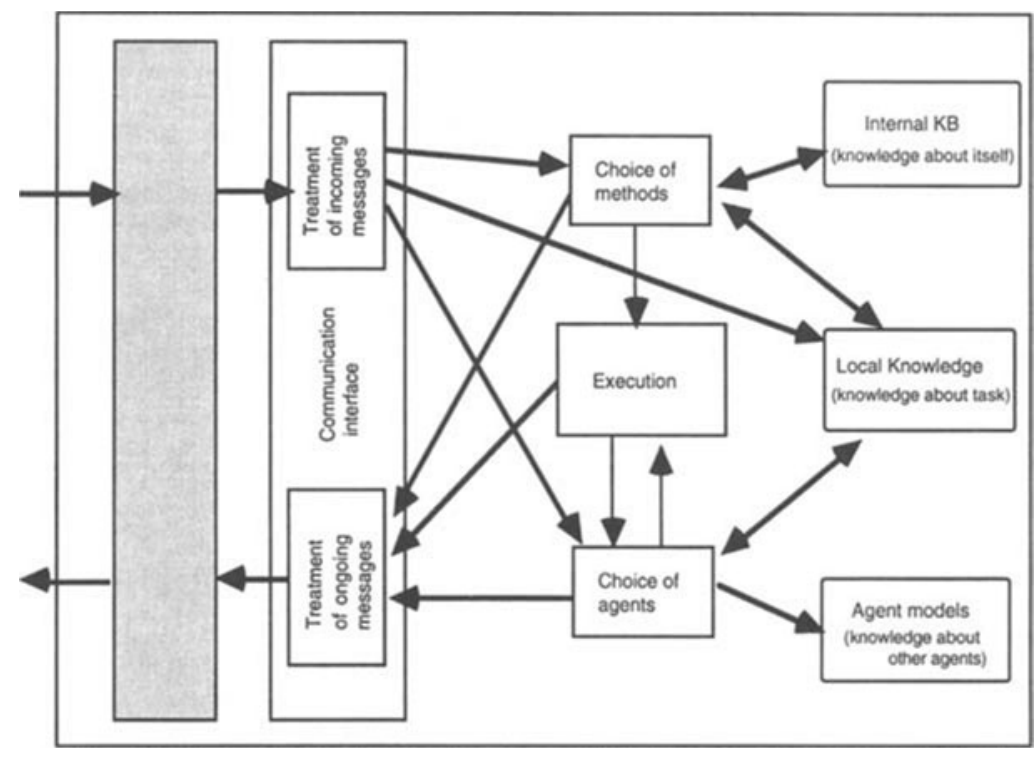

Figure 3 Treatment of message by an agent.

At the beginning of the design or re-design process, the agent Project Manager sends a REQUEST message to the agent Design Representation to ask for defining all necessary objects including classes, instances and methods and storing them in the local database EDBMS or distributed database. If such objects exist in the local database or distributed database, Design Representation sends simply an INFORM message to Project Manager with : content *OK*. If not, Design Representation defines all objects and stores them in the databases by sending NOTICE message to the agent Local $D B$, and then sends an INFORM message to Project Manager. Local $D B$ is a reactive agent. It receives two types of messages: NOTICE messages for storing objects and REQUEST messages for loading objects. In the next paragraphs, we no longer discuss the messages between Local $D B$ and the other agents.

If the project manager desires to design or re-design a gear box, he can specify all the necessary parameters such as the transmission power, input rotational speed and reduction rate and some other constraints by means of a specific interface. The agent Project Manager sends a REQUEST message with initial data to Computational Tool according to its knowledge that Computational Tool can carry out this optimization and then waits for a response. If it does not receive a response for a period which can be known by the knowledge about the agent 
Computational Tool and associated SKILLS, it repeats the REQUEST twice. And if there is still no response, the agent Project Manager considers that Computational Tool is not active and the design task cannot be done at this time. In the current implementation of DIDE, most of agents work on this type of mechanism. In the next paragraphs, we assume that all concerned agents are active and that each agent has decided which agent must be contacted and which SKILLS must be used according to its agent models.

The agent Computational Tool receives the REQUEST message and does the calculation. Upon completion of the calculation, Computational Tool sends an INFORM message with the results to Project Manager. Here the results are some optimized gear box parameters such as gear module, the tooth number of the small gear, or the big gear width.

After receiving this message, the agent Project Manager then sends a REQUEST message to Gear Box Assembly for designing or modifying the gear box. Gear Box Assembly decomposes this design task (which is itself a sub-task of the design project "Gear Box Design") into some sub-tasks and sends multi-cast type REQUEST messages in parallel to three design or selection agents Bolt Assembly Design, Bearing Selection, and Gear Design. These three agents carry out the sub-tasks (selection or calculation) in parallel. During the selection or calculation process, each of these agents first loads the initial object data from local or distributed database, and then modifies them according the standardized or calculated results, and finally stores them into local database and distributed database if all these databases are active. In the current experimental example, each of these agents tries to obtain data from the local database first if they exist, loads them; but if not, it tries to obtain them from the distributed database. The results are stored into two databases whenever they are active. After completing the sub-tasks, each of these three agents sends an INFORM message to Gear Box Assembly. The : content of the INFORM message is *OK* if the sub-task is successfully finished. During the selection and design process in the Bearing Selection and Bolt Assembly Design, the agents need standard tables to obtain standard (nominal) diameter and other standard dimensions of the bearings, the bolts, the nuts or the washers. They send a REQUEST message with a calculated diameter to Database of Engineering Standards and wait for a reply. The agent Database of Engineering Standards receives this type of messages and searches for the requested information in the database of national engineering standards and then sends an INFORM message with the results to requesting agents.

The agent Gear Box Assembly receives the messages from three other design agents, analyses the results and verifies the global constraints. If there are some conflicts, Gear Box Assembly tries to resolve them according to its knowledge. If it cannot resolve some conflicts, it sends a REQUEST message to Project Manager to ask for a decision. After a decision is made, it resends a multi-cast type REQUEST message to the design and selection agents. If there is no conflict among the received results, Gear Box Assembly resumes its work and revises some parameters of the top-case and low-case, such as the diameters of the bolt holes. The object data have to be loaded from the local or distributed database before the verification, and stored into these databases after that verification and modification. And finally, Gear Box Assembly sends an INFORM message to Project Manager with : content *OK*.

After sending a REQUEST message or receiving an INFORM message, each of above mentioned agents stores some important information such as the contents (keyword with initial parameters in the REQUEST message and results in the INFORM message) of the messages in its task model as mentioned in Section 3.4. Afterward, when an agent will send a REQUEST message, firstly it verifies whether it sent a same REQUEST before and had obtained a result. If yes, it does not re-send this REQUEST message, and gets the results directly from its task model.

The Project Manager receives the INFORM message from Gear Box Assembly, may view the design results on a 2D graphical interface developed on LispView, may send a message to Graphical Tooll for displaying the results which can be obtained from the databases, and may also send an E-mail with a http pointer of the IGES file to the production engineer for showing the results on I-DEAS (Graphical Tool2), which will be used for production engineers in future computation. 
The Monitor receives all above messages sent by all other active agents in the same system, displays all received messages on a text interface, shows the current system situation on a graphical interface, and modifies dynamically two HTML files about the agent information and the current situation as mentioned in Section 3.2.

Figure 4. Shows examples of possible message paths among the main autonomous agents of DIDE in the local network.

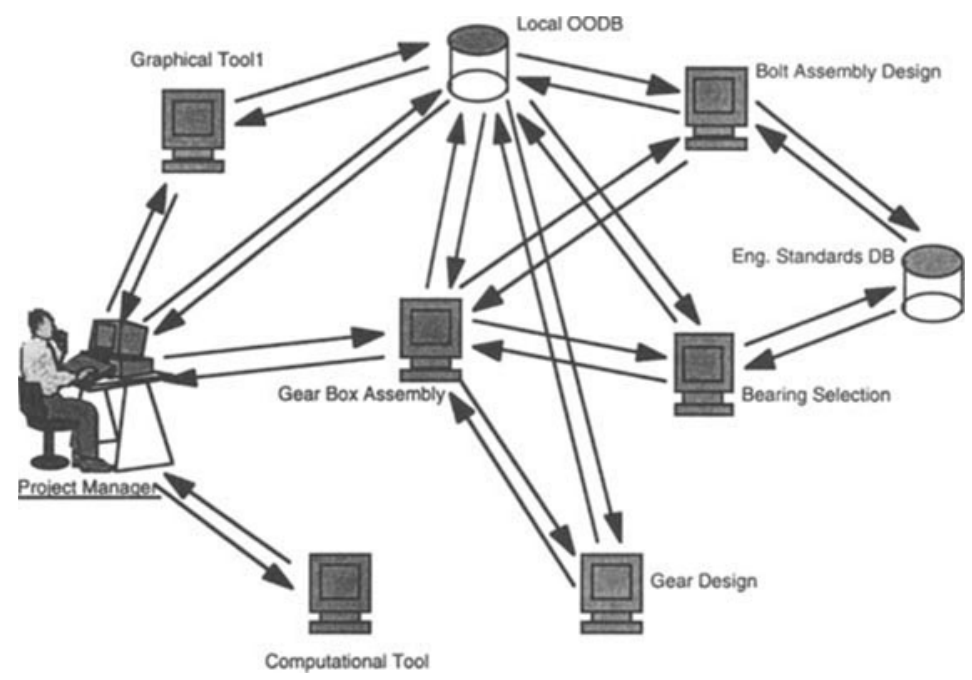

Figure 4 Possible message paths among the main DIDE agents.

\section{CONCLUSION AND DISCUSSION}

The objective of our research is to develop a distributed intelligent design environment for supporting cooperation among the existing engineering tools and human specialists. The techniques of Distributed Artificial Intelligence provide a natural model for such applications. In this paper, we discussed a distributed architecture for an intelligent design environment based upon asynchronous cognitive agents combining a number of mechanisms already found in the literature. Such an architecture is specially useful for large design problems.

We have implemented an experimental design environment using a platform, OSACA, to support the cooperation among the engineering tools organized as independent agents. In this environment, we have successfully implemented communications among about ten independent agents located in the different workstations, which proves that the architecture proposed in this paper is feasible. However, as a result of our experiments in the first version of our implementation, we found that some commercially available products, although usable, are not the best support for this kind of approach. Our goal, as mentioned previously, is to obtain first hand experience in the case of large design projects.

According to our experience, it can be found that multi-agent systems for engineering design problems have some interesting advantages: (i) a multi-agent system can be developed into a real open system, which makes the design system dynamic, i.e., agents can be added and removed as needed without disturbing the working system; (ii) each agent is autonomous and independent, which makes the multi-agent design system less complex than the blackboard system, and also makes it easy to integrate the existing engineering tools into the design system 
so as to resolve the legacy problems; (iii) it is easy to integrate human elements, i.e., it is easy to develop "human agents"-user interface for human specialists; (iv) it makes the design system efficient through parallel executions; ( $v$ ) it is flexible, which means that it is easy to transfer one application to another application by implanting some new agents. However, there are also some drawbacks: (i) it is not easy to build and test multi-agent systems; (ii) it takes much time to establish agent models and local knowledge models (task models), and the inter-agent communication also takes much time, and therefore it is not efficient for small design projects; (iii) there are also some other problems difficult to be solved, such as shared ontologies, or reestablishing temporary consistency in the global system.

The experimental research of the project DIDE produced some useful insights that are applicable in future developments and in other research projects. Here is a brief discussion of some interesting issues based on our experience.

\subsection{Cognitive agent}

An important objective of our research is to design cognitive agents for the DIDE project. A cognitive agent is an agent which has at least the following properties: (i) it is knowledgebased; (ii) it has knowledge about other agents and the knowledge is obtained during the interaction or communication with other agents or learned from a special "tutor" agent; (iii) it is not simply a reactive one, i.e., it does not only reply to the requests from other agents, but it can also give some suggestions or propositions to the current solution according to its knowledge about the current design task. According to our experience, it is feasible to build agents able to memorize previous actions or capable to recognize the task and sub-tasks being tackled. Using artificial intelligence techniques, such agents can be developed to react to certain contexts and criticize or provide some advice to other agents.

\subsection{Agent life cycle}

One of the major problems for developing truly open systems is that of being able to insert or to remove agents on a given application without halting or reinitializing the (distributed) system. Indeed, since large design projects last several months or even several years, new tools or new services appear during the life of the project; similarly, existing tools get upgraded. Thus, it is necessary to accommodate such changes smoothly without disturbing the project. Traditional approaches, in which a group of powerful tools may be integrated into a large, efficient, decision support system, do not allow it. Such approaches are viable only if the problem domain is static, i.e., the tools, design rules, and production process do not change over the product life-cycle. Indeed, when new services are appended to a group of cooperating processes it is usually necessary to recompile all programs on all machines on the network. This is clearly unacceptable.

In the OSACA-based DIDE system agents may appear or disappear with minimal disturbance to the whole system. New agents, whether slaves (e.g., some encapsulated existing tools) or more active, are created from generic agents. They possess communication capabilities and some expertise (which may result from programs, rules, databases, etc.). They are not required to know anything about the existing agents, nor of the ongoing tasks to be solved.

When a new agent connects to the group, it immediately can receive broadcasted messages and respond to them. Other agents are not modified by the introduction of a new agent, i.e., the system keeps running. Once a new agent is connected, it must build its own representation of the task being solved as well as acquire information about the other agents. Currently, two strategies can be used: (i) either a "tutor" agent helps the new agent to do it - this amounted to downloading the information in the MARS project (Abriat 1991); or (ii) models will be build as needed through exchanged messages. Two things could be done in addition: (i) an agent could advertise its skills as soon as it is connected; (ii) an agent could inquire actively once it is connected by broadcasting requests. Such behaviours which can be installed in the generic 
agent from which more specific agents are derived, are not currently implemented in the OSACA agents.

An active agent may die (temporarily or definitively) or be removed from the system. In such a case, generally nothing special is done. In the current DIDE experimental environment, if an agent is normally disconnected, all other agents will note it and modify their agent models accordingly.

\subsection{Agents and Web tools}

The World Wide Web was originally developed as an infrastructure for research collaboration. HTML provides a standard file format for exchanging information among remote agents. Hypertext in HTML can be implemented as special "anchors" that point to resource links to other concerned files from HTTP servers throughout Internet. WWW also includes links to FTP and remote procedure scripts, which can be used to transfer some large files such as DXF or IGES files, and even image or audio-video files in large design projects.

In the DIDE experimental environment, a Web browser, Netscape (or HotJava), has been used to display the information about the active agents and the current situation of the working environment. This is very interesting for cooperation among the design teams located on different sites and communicating via Internet. HTTP has been selected as a main remote communication protocol for DIDE project. According to the experiments in our research group, it is possible to develop agents using Java or Python, so as to use a Web browser like HotJava or Netscape as a user interface for human specialists.

\subsection{Openness and dynamics}

For large engineering design projects, the design process often lasts for a long time, thus, the openness of a system is very important and truly central to the approach. Indeed, it is not easy to let agents join and leave the working system dynamically. Our goal is to verify whether it is actually possible to build truly open systems, that is, systems for which users can freely add or remove agents without having to halt or to reinitialize the work in progress in any way. The OSACA-based DIDE environment implements this idea.

Here we give an example of a situation that we encountered during experimental stages, showing the openness and the dynamics of our system. During the bolt assembly design process, Bolt Assembly Design sends a REQUEST message to Database of Engineering Standards for obtaining standard bolt length and bolt thread length. Database of Engineering Standards received the messages and called the corresponding functions (skills) to search standard data. But there was a problem in the function GETSTTL (for searching standard bolt thread length) and this agent was out of order and became non-active. We had to "repair" this agent by redefining this function and then re-start the agent. Afterward, the agent continued to receive REQUEST messages, dealt with the received messages and sent out the results without disturbing the whole working environment. Bolt Assembly Design waited for results from Database of Engineering Standards for several minutes without response and subsequently repeated the REQUEST message twice. This mechanism allowed Database of Engineering Standards to receive the REQUEST message from Bolt Assembly Design and to complete the design process.

\subsection{Communication}

Communication is the key issue in distributed systems. Indeed, the complexity of building a single system has been traded for a reduced complexity of each agent. However, the difficulty has been transferred in part to the level of communications and to the issue of combining the separately achieved sub-tasks.

Communication can be implemented in very different ways, depending on the nature of the agents, the global architecture of the system, the timing of the exchanges, or the number of 
receivers for a message. Indeed, communications can occur between humans and/or machines; they can be direct by means of messages or indirect by posting; they can be synchronous or asynchronous; they can concern a single agent or several agents.

Communication can be direct or indirect. In multi-agent systems communication is directly done between agents by using explicit messages. In multi-expert systems, communication is done indirectly by writing to the blackboard (which, however, is equivalent to a multicast communication strategy in the case of partitioned blackboards; broadcast otherwise). In the latter case, the communication mechanisms can be quite simple, like the SHARED workspace (Wong and Sriram 1993) in the DICE projects.

Communication can be synchronous or asynchronous. Even if synchronous communications (blackboard style) are very useful for systems in which the cooperating agents have to work simultaneously, such as a multimedia teleconferencing system (Lee, Mansfield, and Sheth 1993), we found that they are not necessary for the type of the design work we are considering because we would like to develop environments for large design problems, for which the design period is often very long and synchronous communications in this case are neither required, nor efficient or economical.

\subsection{Shared ontologies}

An active agent system works by exchanging messages at a high semantic level. This raises the question of shared vocabulary and common ontologies, and also of the initial expert knowledge content of each agent prior to its connection to the network. A review of such questions can be found in (Cavalli et al 1991) in the context of "Enterprise Integration" and in (Gruber 1993) in the context of "Ontologies and knowledge sharing." Our approach in this domain is to manually organize needed concepts into minimal ontologies as needed. Then, whenever a new agent enters the system, it is its responsibility to make sure that the system contains all the needed ontologies. If not, it must bring the missing ontologies.

\subsection{Conflict resolution}

Conflicts occur in multidisciplinary design environments mainly for two reasons: individual participants in the cooperative process lack the complete knowledge of global objectives necessary to ensure that their locally-preferred solutions are in alignment with these higher objectives, and individual disciplines have individual ideas about what constitutes the best design. Even individual design requires trade-offs because of competing design criteria, such as safety, cost and social acceptance, as well as artefact requirements and specifications. The ability of designers to avoid or minimize conflicts through judicious trade-offs and other methods is one of their most valuable skills.

Resolution and detection of conflicts are especially difficult when the design task as well as knowledge concerning such competing factors is distributed among different actors with different perspectives. Certain methods such as negotiation, hierarchical structures, constraint weakening, creation of new variables and user interaction can be used for conflict resolution. It is also possible to combine several methods in the same system.

Researchers have developed conflict resolution strategies for various types of conflicts: introducing an approach to conflict resolution based on classifying the conflict and mapping it to a specific strategy (Klein 1991); resolving conflicts in resources allocation among cooperative agents (Conry, Meyer, and Lesser 1995); resolving conflicts via negotiation (Sycara 1990; Chu-Carroll and Carberry 1995; Dunskus et al 1995; Bahler, Dupont, and Bowen 1994); assisting the designer by using expert systems that offer criticisms and suggestions concerning design decisions in SNEAKERS (Douglas, Brown, and Znger 1993). Two interesting examples can be found in the project NEXT-LINK (Petrie et al 1994) and in the project SHARED-DRIMS (Pena, Sriram, and Logcher 1993). NEXT-LINK uses a simple and ubiquitous notion of constraint to represent all conflicts and provides a support for humanguided conflict resolution. On the contrary, SHARED-DRIM uses an automatic conflict 
resolution technology for resolving the known conflicts when solutions are available in its knowledge base.

In DIDE, we leave each group of designers develop possible conflicting partial solutions (divergence) (Barthès 1993). Such solutions are managed as parallel versions. Then, at the review meetings all groups have to compromise on a commonly agreed solution or on a solution imposed by the project manager (reconciliation). This relates to the global consistency of the project and not to the consistency within each partial solution, which we have not yet addressed (see also Project Manager in the Section 4.2.2).

DIDE is an ongoing research project. Some important issues are being studied and developed: remote communication, conflict detection and resolution, and shared ontologies.

\section{REFERENCES}

Abriat, P. (1991) Conception et réalisation d'un système multi-agent de robotique permettant de récupérer les erreurs dans les cellules flexibles. Thèse de Doctorat, Université de Technologie de Compiègne.

Bahler, D.; Dupont, C.; and Bowen, J. (1994) Anaxiomatic approach that supports negotiated resolution of design conflicts in concurrent engineering. Artificial Intelligence in Design, Gero, J.S. and Sudweeks, D. (eds.), Kluwer Academic Publishers, Dordrecht, pp 363379.

Balasubramanian, S. and Norrie, D.H. (1995) A Multi-Agent Intelligent Design System Integrating Manufacturing and Shop-Floor Control. In Proceedings of First International Conference on Multi-Agent Systems, San Francisco, USA, The AAAI press / The MIT Press.

Barbuceanu, M. and Fox, M. (1995) COOL: A Language for Describing Coordination in Multi Agent Systems. In Proceedings of First International Conference on Multi-Agent Systems, San Francisco, USA, The AAAI press / The MIT Press.

Barthès, J.P. (1989) MOSS: a multi-user object environment. In Proceeding of 2nd Symposium on AI, Monterrey, Mexico.

Barthès, J.P. (1993) La problèmatique de reconciliation en ingénierie simultanée. In Actes 01 DESIGN'93, Tunis.

Barthès, J.P. (1995) Do we have the technology for supporting knowledge intensive CAD in large design projects? Knowledge Intensive CAD-1, Tomiyama, T.; Mantyla, M. and Finger, S. (editors), Espoo, Finland.

Boissier, O. (1993) Problème du contrôle dans un système intégré de vision: Utilisation d'un système multi-agent. In Actes de la lère journées francophones Intelligence Artificielle Distribuée et Système Multi-Agents, Toulouse, France.

Brandt, W.D. and Petro, J.J. (1992) Integrating the CE Approach of the CERC into Engineering Scenarios", CERC Technical Report, CERC-TR-TM-92-005, West Virginia University, WV, USA.

Branki, N.E. and Bridges, A. (1993) An Architecture for Cooperative Agents and Applications in Design", In Advanced Technologies, Beheshi, M.R. and Zreik, K., Eds., pp. 221230.

Brown, D.C.; Dunskus, B.; Grecu, D.L. and Berker, I. (1995) SINE: Support For Single Function Agents, in AIENG'95, Applications of AI in Engineering, Udine, Italy.

Cavalli, A.; Hardin, J.; Petrie, C.; Smith, R.; and Speyer, B. (1991) Technical Issues of Enterprise Integration, Research Report EID-349-91, MCC, Austin, Texas.

Chu-Carroll, J. and Carberry, S. (1995) Communication for conflict resolution in Multi-Agent Collaborative Planning. In Proceeding of the First International Conference on MultiAgent Systems, San Francisco, USA, The AAAI press/The MIT press, pp 49-56. 
Cockburn, D. and Jennings, N.R. (1995) ARCHON: A DAI System for Industrial Applications. Foundations of DAI, eds. G.M.P.O'Hare and Jennings, N.R., Wiley Interscience.

Conry, S.E.; Meyer, R.A.; and Lesser, V.R. (1988) Multistage negotiation in distributed planning. Readings in Distributed Artificial Intelligence, Bond, A.H.; and Gasser, L., eds., Morgan Kaufmann Pulishers, Inc. pp 367-383.

Coyne, R.; Finger, S.; Konda, S.; Prinz, F.B.; Sievorek, D.P.; Subrahmanian, E.; Tenenbaum, M.J. (1994) Creating an advanced collaborative open resource network. In Proceeding of the sixth International ASME Conference on Design Theory and Methodology, Minneapolis, MN, USA.

Cutkosky, M.R.; Engelmore, R.S.; Fikes, R.E.; Genesereth, M.R.; Gruber, T.R.; Mark, W.S.; Tenenbaum, J.M.; and Weber, J.C. (1993) PACT: An Experiment in Integrating Concurrent Engineering Systems. IEEE Computer, 26(1), 28--37.

Demazeau, Y. (1993) La plate-forme PACO et ses applications. In Actes de la 2ème Journée Nationale du PRC-IA sur les Systèmes Multi-Agents, Montpellier, France.

Douglas, R.E.; Brown, D.C. and Znger, D.C. (1993) A Concurrent Engineering Demonstation and Training System for Engineers and Managers. Internationa Journal of CAD/CAM and Computer Graphics, special issue, (ed) Costea, I., Hermes, 8(3), 263-301.

Dunskus, B.; Grecu, D.L.; Brown, D.C. and Berker, I. (1995) Using Single Function Agents to investigate Negotiation, AIEDAM: Artificial Intelligence for Engineering Design, Analysis and Manufacturing, Special Issue: Conflict Management in Design, Smith, I., Ed., Cambridge U.P.

Fenves, S.J.; Flemming, U.; Hendrickson, C.; Maher M.L.; and Schimitt, G. (1990) An Integrated Software Environment for Building Design and Construction, Computer-Aided Design, 22(1), 27-36.

Finin, T.; McKay, D.; and Fritzson, R. (1992) Specification of the KQML: AgentCommunication Language, Tech. Report EIT TR 92-04, Enterprise Integration Technologies, Palo Alto, Calif., USA.

Frankel, R. (1991) The ToolTalk Service, A Technical Report, SunSoft.

Gero, J.S. (1987) Expert systems in computer-aided design, Elsevier Science Publishers, Palo Alto, Cali., USA.

Grecu, D.L. and Brown, D.C. (1995) Design Agents That Learn. IJCAI-95 Workshop on Adaptation and Learning in Multiagent Systems, Montreal, Canada.

Gruber, T. (1993) A Translation Approach to Portable Ontology Specification. Knowledge Acquisition, 5(2), 199--220.

Iffenecker, C. (1994) Modélisation et réutilisation d'expertises variées en Conception. In Actes du Séminaire Interdisciplinaire "Mémoire Collective", Compiègne, France.

Jennings, N.R.; Corera, J.M. and Laresgoiti, I. (1995) Developing Industrial Multi-Agent Systems. In Proceedings of First International Conference on Multi-Agent Systems, San Francisco, USA, The AAAI press / The MIT Press.

Jones, M.; Wheadon, J.; Whitgift, D.; Niezette, M.; Timmermans, R.; Rodriguez, I.; Romero, R. (1995) An agent based approach to spacecraft mission operations. In Towards Very Large Knowledge Bases, Mars, N., Ed., IOS Press, Amsterdam.

Klein, M. (1991) Supporting Conflict Resolution in Cooperative Design Systems. IEEE Trans. Systems, Man \& Cyb., 21(6).

Lee, K.C.; Mansfield, W.H.Jr.; and Sheth, A.P. (1993) A Framework for Controlling Cooperative Agents. IEEE Computer, 26(7), 8--16.

Londono, F.; Cleetus, K.J.; and Reddy, Y.V. (1990) A Blackboard Scheme for Cooperative Problem-Solving by Human Experts, in Sriram,D.; Logcher, R.; and Fukuda, S. (eds), Computer-Aided Cooperative Product Development, Springer, pp 26-50.

Mantyla, M. (1995) Product modelling for knowledge intensive CAD - towards a research agenda. Knowledge Intensive CAD-1, Tomiyama, T.; Mantyla, M. and Finger, S. (editors), Espoo, Finland. 
McGuire, J.M.; Kuokka, D.R.; Weber, J.C.; Tenenbaum, J.M.; Gruber, T.R. and Olsen, G.R. (1993) SHADE: Technology for Knowledge-Based Collaborative Engineering, Journal of Concurrent Engineering: Application and Research, 1(3).

Monceyron, E. and Barthès, J.P. (1992) Architecture for ICAD Systems: an Example from Harbor Design. Revue Science et Techniques de la Conception, 1(1), 49--68.

Morse, D.V. (1990) Communication in Automated Interactive Engineering Design. PhD thesis, Carnegie Mellon University.

Papazoglou, M.P.; Laufmann, S.C.; and Sellis T.K. (1992) An Organizational Framework for Cooperating Intelligent Information Systems. International Journal of Intelligent and Cooperative Information Systems, 1(1), 169-202.

Park, H.; Cutkosky, M.R.; Conru, A.B.; and Lee, S.H. (1994) An Agent-Based Approach to Concurrent Cable Harness Design. AIEDAM, 8(1).

Pena, F.; Sriram, D.; and Logcher, R. (1993) SHARED-DRIMS: SHARED Design Recommendation-Intent Management System, In the Proceedings of 2nd IEEE Workshop on Enabling Technologies Infrastructure for Collaborative Enterprises (WET ICE).

Petrie, C.; Cutkosky, M.; Webster, T.; Conru, A. and Park, H. (1994) Next-Link: An Experiment in Coordination of Distributed Agents. Position paper for the AID-94 Workshop on Conflict Resolution, Lausanne.

Populaire, Ph.; Demazeau, Y.; Boissier, O.; and Sichman, J. (1993) Description et implémentation de protocole de communication en univers multi-agents. In Actes de la lère journées francophones Intelligence Artificielle Distribuée et Système Multi-Agents, Toulouse, France.

Quadrel, R.W.; Woodbury, R.F.; Fenves, S.J.; and Talukdar, S.N. (1993) Controlling Asynchronous Team Design Environments by Simulated Annealing. Research in Engineering Design, 5(2), 88--104.

Reddy, R.Y.V.; Srinivas, K.; Jagannathan, V.; and Karinthi, R. (1993) Computer Supported for Concurrent Engineering. IEEE Computer, 26(1): 12-16.

Reddy, J.M.; Chan, B. and Finger, S. (1995) Patterns in design discours: A case study. Knowledge Intensive CAD-1, Tomiyama, T.; Mantyla, M. and Finger, S. (editors), Espoo, Finland.

Ribeiro Gouveia, F. and Barthès, J.P. (1993) Cooperative Agents in Engineering Environments. In Proceedings of EuropIA'93 Workshop on Intelligent Information Environments, Delft.

Saad, M. and Maher, M.L. (1996) Shared understanding in computer-supported collaborative design, Computer-Aided Design, 28(3), 183-192.

Scalabrin, E. and Barthès, J.P. (1993) OSACA, une architecture ouverte d'agents cognitifs indépendants. In Actes de la 2ème Journée Nationale du PRC-IA sur les Systèmes MultiAgents, Montpellier, France.

Shen, W. and Barthès, J.P. (1995a) An object-oriented approach to engineering design product modelling. Knowledge Intensive CAD-1, Tomiyama, T.; Mantyla, M. and Finger, S. (editors), Espoo, Finland.

Shen, W.; Barthès, J.P. (1995b) DIDE: A Multi-Agent Environment for Engineering Design. In Proceeding of The First International Conference on Multi-Agent Systems, San Francisco, USA, The AAAI press / The MIT Press.

Sriram, D.; Stephanopoulos, G.; Logcher, R.; Gossard, D.; Groleau, N.; Serrano, D.; and Navinchandra, D. (1989) Knowledge-based Systems. Applications in Engineering Design: Research at MIT. AI Magazine, 10(3): 79-96.

Sriram, D.; Logcher, R.; Wong, A.; and Ahmed, S. (1991) An object-oriented framework for Collaborative Engineering Design. Lecture Notes in Computer Science, pp.51-92. Springer-Verlag.

Sriram, D.; Logcher, R.; Groleau, N.; and Cherneff, J. (1992) DICE: An Object Oriented Programming Environment for Cooperative Engineering Design. AI in Engineering Design Vo.3, Tong C. and Sriram D. (Eds.), Academic Press. 
Stephanopoulos, G.; Johnston, J.; Kriticos, T.; Lakshmanan, R.; Mavrovouniotis, M.; and Siletti, C. (1987) DESIGN-KIT: An Object-Oriented Environment for Process Engineering. Computer in Chemical Engineering 11(6).

Sycara, K.P. (1990) Cooperative Negotiation in Concurrent Engineering Design. Cooperative Engineering Design, Springer Verlag.

Thoraval, P.; and Mazas, Ph. (1990) ARCHIX, an Experiment with Intelligent Design in the Automobile Industry. In Proceeding of 4th Eurographics Workshop on Intelligent CAD Systems, Mortefontaine, France.

Tomiyama, T.; Umeda, Y.; Ishii, M.; Yoshioka, M.; and Kiriyama, T. (1995) Knowledge systematization for a knowledge intensive engineering framework. Knowledge Intensive $C A D-1$, Tomiyama, T.; Mantyla, M. and Finger, S. (editors), Espoo, Finland.

Tou, I.; Berson, S.; Estrin, G.; Eterovic, Y.; and Wu, E. (1994) Prototyping Synchronous Group Applications. IEEE Computer, 27(5), 48-56.

Toye, G.; Cutkosky, M.; Leifer, L.; Tenenbaum, J.; and Glicksman, J. (1993) SHARE: A Methodology and Environment for Collaborative Product Development. In Proceeding of 2nd Workshop on Enabling Technologies: Infrastructure for Collaborative Enterprises, IEEE Computer Press, pp.33-47.

Trousse, B. (1993) Towards a multi-agent approach for cooperative distributed design assistants. In Proceeding of EuropIA'93 Workshop on Intelligent Information Environments, Delft.

Van Dyke Parunak H. (1990) Toward a formal model of inter-agent control. In Proceeding of 10th AAAI Int'l Workshop on Distributed Artificial Intelligence, Bandera, TX.

Wong, A. (1993) Shared Workspace for Collaborative Engineering, $\mathrm{PhD}$ Thesis, Intelligent Engineering Systems Laboratory, MIT.

Wong, A. and Sriram, D. (1993) SHARED: An Information Model for Cooperative Product Development, Research in Engineering Design, 5(1), 21-39.

\section{BIOGRAPHY}

Weiming Shen obtained his BS and MS in Mechanical Engineering from Northern Jiaotong University, Beijing, P.R. China in 1983 and 1986. He worked at Northern Jiaotong University as a lecturer in the department of mechanical engineering from 1986 to 1992 and was appointed as the vice-director of CAD/CAM Laboratory by the university in 1989. Currently he is $\mathrm{PhD}$ Student at University of Technology of Compiegne, France, and will graduate in December 1996. His research interests are design-knowledge representation, object-oriented technology, distributed artificial intelligence and its applications in concurrent engineering.

Jean-Paul A. Barthès obtainted his PhD at Stanford University in 1973. Currently he is a professor in the department of computer science at the University of Technology of Compiègne, and is in charge of the research in Artificial Intelligence. His main interest lies in distributed artificial intelligence (societies of cognitive agents) with applications to the domain of concurrent engineering. He is also the president of IIIA, an institute hosting a European industrial program concerned with the problems of capitalizing industrial and corporate knowledge. 